\title{
Solving the Unit Commitment Problem in Power Generation by Primal and Dual Methods*
}

\author{
D. Dentcheva ${ }^{1}$, R. Gollmer ${ }^{1}$, A. Möller ${ }^{1}$, \\ W. Römisch ${ }^{1}$ and R. Schultz ${ }^{2}$
}

\begin{abstract}
The unit commitment problem in power plant operation planning is addressed. For a real power system comprising coal- and gas-fired thermal and pumped-storage hydro plants a large-scale mixed integer optimization model for unit commitment is developed. Then primal and dual approaches to solving the optimization problem are presented and results of test runs are reported.
\end{abstract}

\section{Introduction}

The unit commitment problem in electricity production deals with the fuel cost optimal scheduling of on/off decisions and output levels for generating units in a power system over a certain time horizon. The problem typically involves technological and economic constraints. Depending on the shares of nuclear, conventional thermal, hydro and pumped-storage hydro power in the underlying generation system fairly different cost functions and side conditions arise in mathematical models for unit commitment. In the present paper we consider a power system comprising coaland gas-fired thermal units and pumped-storage hydro plants. This reflects the energy situation in the eastern part of Germany. Our work grew out of a cooperation with the power company VEAG Vereinigte Energiewerke AG Berlin.

In our unit commitment model, the objective function is given by start-up and operation costs of the thermal units. Pumped storage plants do not cause direct fuel costs. Their operation, nevertheless, has an impact on the total fuel costs in the system. Constraints of the unit commitment model comprise output bounds for the units of the generation system, load coverage over the whole time horizon as well as provision for a spinning reserve, minimum down times for thermal units and water balances in the pumped-storage plants. Typical optimization horizons vary from several days up to several months.

From the extensive literature in unit commitment we mention here [1], [3], [5], [6], [12], [18], and [19], and refer to the comprehensive literature synopsis [17]. The papers [6], [10], [18] reflect recent developments of efficient algorithms based on modern mathematical techniques.

${ }^{*}$ This research is supported by a grant of the German Federal Ministry of Education, Science, Research and Technology (BMBF).

${ }^{1}$ Humboldt-Universität Berlin, Institut für Mathematik, 10099 Berlin

${ }^{2}$ Konrad-Zuse-Zentrum für Informationstechnik Berlin, Heilbronner Straße 10, 10711 Berlin 


\section{Modelling}

Our mathematical model for the unit commitment problem is a mixed-integer optimization problem with linear constraints. In the following sections we will tackle this problem with both primal and dual solution methods, which will lead to different model specifications and variants. Here, we describe features of the model that are common to both situations.

The optimization horizon is partitioned into (hourly, half-hourly or shorter) time intervals, whose total number is denoted by $T$. By $I$ and $J$ we denote the number of thermal and pumped-storage hydro units in the system, respectively. Then the following variables occur

$$
\begin{aligned}
& \boldsymbol{u}_{i}^{t} \in\{0,1\} \quad \text { - on/off decisions for the thermal unit } i \in\{1, \ldots, I\} \\
& \text { and time interval } t \in\{1, \ldots, T\} \text {, } \\
& \boldsymbol{p}_{i}^{t} \quad \text { - output level for the thermal unit } i \text { during interval } t \text {, } \\
& \boldsymbol{s}_{j}^{t}, \boldsymbol{w}_{j}^{t} \quad \text { - level for generation and pumping, respectively, for } \\
& \text { the pumped-storage plant } j \in\{1, \ldots, J\} \text { during } \\
& \text { interval } t \text {, } \\
& \boldsymbol{l}_{j}^{t} \quad \text { - water level, in terms of energy, in the upper reservoir } \\
& \text { of plant } j \in\{1, \ldots, J\} \text { at the end of interval } t \text {. }
\end{aligned}
$$

The objective function reads

$$
F(\boldsymbol{u}, \boldsymbol{p}):=\sum_{t=1}^{T} \sum_{i=1}^{I} F C_{i}\left(\boldsymbol{p}_{i}^{t}, \boldsymbol{u}_{i}^{t}\right)+\sum_{t=1}^{T} \sum_{i=1}^{I} S C_{i}\left(\boldsymbol{u}_{i}(t)\right) .
$$

Here, $F C_{i}$ denotes the fuel cost function for the operation of the $i$-th thermal unit. With respect to $\boldsymbol{p}_{i}^{t}$ the function is monotonically increasing and often assumed to be convex (linear, piecewise linear, quadratic). Non-convex setups for fuel costs are not considered in the present paper (for their discussion we refer to [14]). Start-up $\operatorname{costs} S C_{i}\left(\boldsymbol{u}_{i}(t)\right)=S C_{i}\left(\boldsymbol{u}_{i}^{t}, \ldots, \boldsymbol{u}_{i}^{t_{s}}\right)$ of the $i$-th thermal unit are determined by the preceding down-time $t-t_{s_{i}}$ and will be further specified later on.

The constraints of the unit commitment model are formulated as linear equations and inequalities. The same feasible set can be described by nonlinear expressions, too. Here, we prefer the linear variant in order to apply methodology from mixedinteger linear programming if also the objective is given by linear terms.

All variables mentioned above have finite lower and upper bounds which reflect the bounded output of all units in the generation system.

$$
\begin{aligned}
p_{i t}^{\text {min }} \boldsymbol{u}_{i}^{t} & \leq \boldsymbol{p}_{i}^{t} \leq p_{i t}^{\max } \boldsymbol{u}_{i}^{t}, i=1, \ldots, I, t=1, \ldots, T \\
0 & \leq \boldsymbol{s}_{j}^{t} \leq s_{j t}^{\max }, \quad j=1, \ldots, J, t=1, \ldots, T \\
0 & \leq \boldsymbol{w}_{j}^{t} \leq w_{j t}^{\max }, \quad j=1, \ldots, J, t=1, \ldots, T
\end{aligned}
$$

The constants $p_{i t}^{\min }, p_{i t}^{\max }, s_{j t}^{\max }, w_{j t}^{\max }$ denote the minimal and maximal outputs, respectively.

Load coverage for each time interval $t$ of the optimization horizon leads to the equations

$$
\sum_{i=1}^{I} \boldsymbol{p}_{i}^{t}+\sum_{j=1}^{J}\left(\boldsymbol{s}_{j}^{t}-\boldsymbol{w}_{j}^{t}\right)=D^{t}, t=1, \ldots, T,
$$


where $D^{t}$ denotes the load to be covered in time interval $t$. Sudden load increases or unforeseen conditions (e.g. outage of a unit) have to be compensated on-line. Therefore, for each time interval $t$, some spinning reserve $R^{t}$ in the termal units is required which leads to the constraints

$$
\sum_{i=1}^{I}\left(p_{i t}^{\max } \boldsymbol{u}_{i}^{t}-\boldsymbol{p}_{i}^{t}\right) \geq R^{t}, t=1, \ldots, T .
$$

During the whole optimization horizon water balances in the pumped-storage plants have to be maintained. It is typical of the power system of VEAG that no additional in- or outflows arise in the upper reservoirs of the pumped-storage plants such that these operate with a constant amount of water. The possible workload for turbines and pumps is restricted by the water levels and capacities of the upper reservoirs. This is modelled by the inequalities

$$
0 \leq \boldsymbol{l}_{j}^{t} \leq l_{j}^{\max }, \quad j=1, \ldots, J, t=1, \ldots, T
$$

where $\boldsymbol{l}_{j}^{t}$ is connected with the variables $\boldsymbol{s}_{j}^{t}$ and $\boldsymbol{w}_{j}^{t}$ by the reservoir constraints

$$
\begin{aligned}
& \boldsymbol{l}_{j}^{t}=\boldsymbol{l}_{j}^{t-1}-\boldsymbol{s}_{j}^{t}+\eta_{j}^{t} \boldsymbol{w}_{j}^{t}, \quad t=1, \ldots, T, \\
& \boldsymbol{l}_{j}^{0}=l_{j}^{i n i}, \boldsymbol{l}_{j}^{T}=l_{j}^{\text {end }}, \quad j=1, \ldots, J
\end{aligned}
$$

Here $l_{j}^{\text {ini }}, l_{j}^{\max }$ and $l_{j}^{\text {end }}$ denote the initial, maximal and terminal water levels in the upper reservoir, and $\eta_{j}$ is the efficiency of the $j$-th pumped-storage plant.

Finally, there are minimal down times $\tau_{i}$ for the thermal units that are mainly determined by technological reasons and serve to avoid erosion of the unit by too frequent changes of thermal stress.

$$
\begin{aligned}
\boldsymbol{u}_{i}^{t-1}-\boldsymbol{u}_{i}^{t} \leq 1-\boldsymbol{u}_{i}^{l}, \quad l & =t+1, \ldots, \min \left\{t+\tau_{i}-1, T\right\}, i=1, \ldots, I \\
t & =2, \ldots, T-\tau_{i}+1 .
\end{aligned}
$$

\section{Primal Methods}

Our primal approach to the unit commitment problem relies on solving the underlying large-scale mixed-integer optimization problem by adapted branch-and-bound techniques, possibly enriched by cutting planes derived from the convex hull of feasible points. In order to apply the corresponding methodology and implementations we formulate the basic model from Section 2 as a mixed-integer linear program. More precisely, we assume that, for each thermal unit $i \in\{1, \ldots, I\}$, the fuel costs are affine-linear functions of the generated power, and the start-up costs are given by

$$
S C_{i}\left(\boldsymbol{u}_{i}(t)\right)=A_{i} \max \left\{\boldsymbol{u}_{i}^{t}-\boldsymbol{u}_{i}^{t-1}, 0\right\}, t=2, \ldots, T
$$

where $A_{i}$ is some positive constant. This piecewise linear convex function can be expressed in linear terms by introducing an additional variable and adding two linear inequalities to the constraints.

The setups for fuel as well as start-up costs are the simplest possible one could think of. On the other hand, the real-life data material from VEAG, by means of which we 
have validated our models, indicates that both model simplifications are tolerable. Staying within the framework of a linear model it is possible to refine the linear fuel costs by a piecewise linear setup and to take into account that startup costs vary with the preceding down-time of the unit. The tradeoff for both extensions is a growing number of variables and constraints in the programs to be solved.

It is a typical feature of the generation system of VEAG that there are thermal units which are absolutely identical. Therefore, obviously, a model reformulation is possible, treating each group of identical blocks by only one status variable $\boldsymbol{u}_{i}^{t}$ and one output variable $\boldsymbol{p}_{i}^{t}$. The status variable now is not a Boolean one but with the number of identical blocks being $N$ we would have $\boldsymbol{u}_{i}^{t} \in\{0, \ldots, N\}$, indicating the number of blocks in on-state. The generalized lower and upper bounds (2.2) for the output $\boldsymbol{p}_{i}^{t}$ of the group of units remain valid and so does the formulation of start-up costs.

In our test runs we have tried both models. The first model corresponds to the pure Boolean setting, the second one to the modification described in the previous paragraph. Though the reduction of the number of integer variables is only by a factor of 0.3 , we observe a considerable reduction of the branch-and-bound tree and the computing times. From this point of view the latter model is superior. On the other hand, the development towards a branch-and-cut algorithm on the basis of valid inequalities developed in [16] relies on a Boolean structure in the form of a knapsack problem and thus could be carried on for the first model only. Further investigation is necessary to decide whether the possible improvement of bounds by the cuts or the reduction of the number of integer variables by the model reformulation are preferable.

The test runs proved the successful applicability of the primal approach to real-life models of the type outlined above. Our program was developed on the basis of modules from the CPLEX Callable Library [4]. The details of the runs are given in Table 1. We used three parks of generating units, reflecting three different states of development. Park 1 consists of 20 thermal units and 6 pumped-storage hydro plants; park 2 has two additional coal-fired units and a modified pumped-storage unit. Park 3 comprises another three additional coal-fired units and one additional pumped-storage unit. Computations were done for three selected weeks ( 8 days) with 1-hour-intervals $(T=192)$ on a HP-Apollo $735 / 125$.

\begin{tabular}{|cc|c|c|c|c|c|c|}
\hline \multicolumn{2}{|c|}{ variant } & \multicolumn{2}{c|}{ park 1 } & \multicolumn{2}{c|}{ park 2 } & \multicolumn{2}{c|}{ park 3 } \\
\cline { 3 - 8 } week & model & time & quality & time & quality & time & quality \\
\hline \hline holiday & 1 & $5: 13$ & $0.164 \%$ & $6: 35$ & $2.965 \%$ & $29: 45$ & $2.837 \%$ \\
week & 2 & $2: 13$ & $0.192 \%$ & $3: 50$ & $0.002 \%$ & $5: 20$ & $0.906 \%$ \\
\hline low & 1 & $6: 09$ & $0.399 \%$ & $17: 26$ & $2.151 \%$ & $21: 59$ & $1.269 \%$ \\
load & 2 & $2: 47$ & $0.425 \%$ & $7: 14$ & $1.152 \%$ & $7: 55$ & $2.929 \%$ \\
\hline peak & 1 & $0: 01$ & $-1)$ & $3: 42$ & $0.086 \%$ & $5: 16$ & $0.005 \%$ \\
load & 2 & $0: 01$ & $-1)$ & $1: 53$ & $0.099 \%$ & $2: 48$ & $0.269 \%$ \\
\hline
\end{tabular}

1) No feasible solution exists.

Table 1: CPU-time in minutes (HP-Apollo 735/125) and upper bound for the deviation of the objective value from the optimum 
The figure shows the load, the thermal generation and the use of pumped-storage units for park 2 in the peak load week.

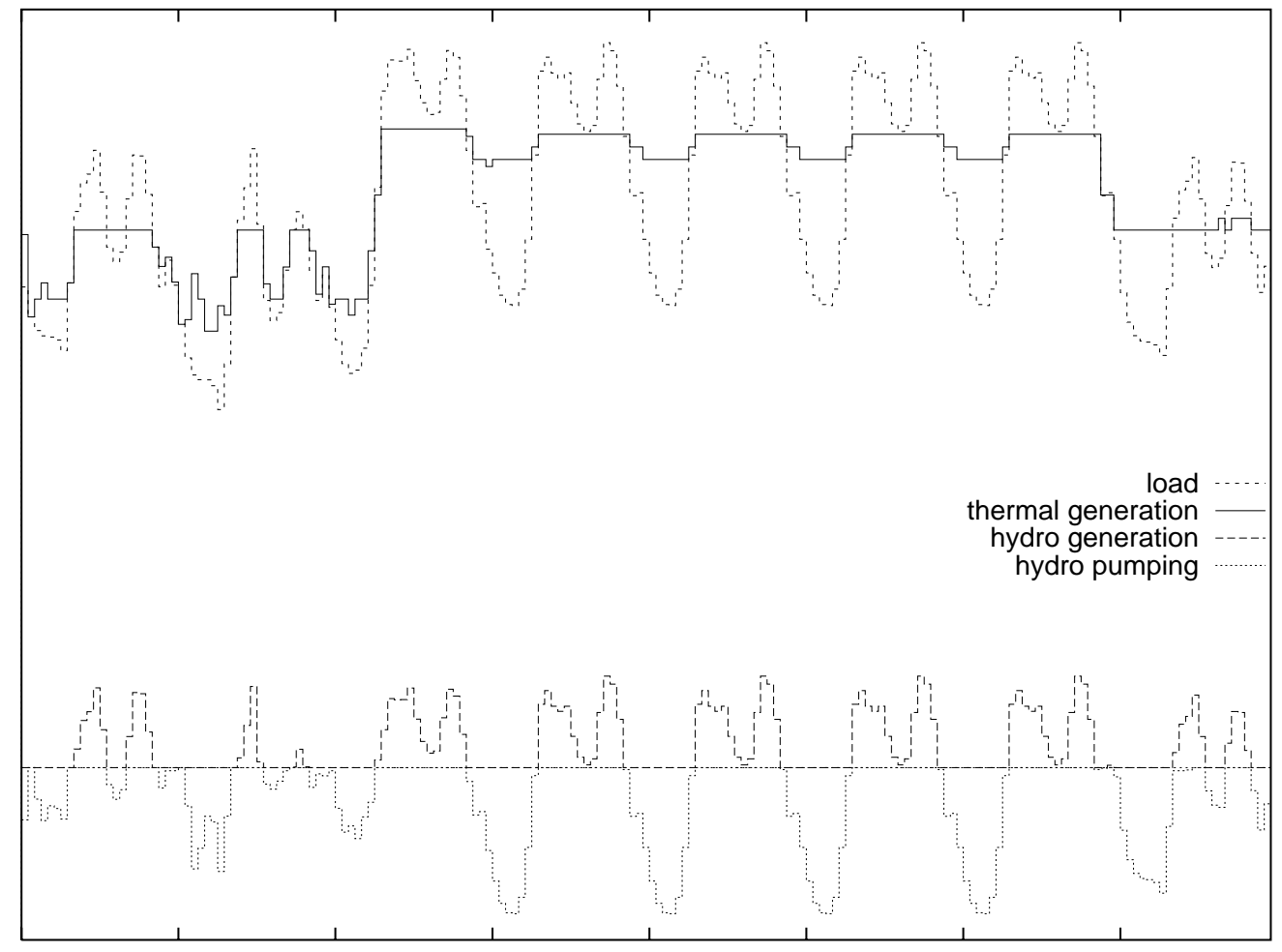

Fig. 1: schedule for park 2 in the peak load week

\section{Dual approach}

The dual approach to the unit commitment problem has been widely studied in the last 15 years (see [17]). In [13], the authors have addressed unit commitment with Lagrangian relaxation for the first time. The main idea is to incorporate the loosely coupling constraints linking operation of different units into the objective function by use of Lagrange multipliers. Then the problem decomposes into smaller subproblems. The solution of the relaxed problem provides a lower bound on the optimal solution of the original problem. The value of the lower bound is a function of the Lagrangian multipliers. This approach has found a wide application for large systems, for two reasons. It works fast due to the decomposition of the dual problem into essentially smaller subproblems. On the other hand, it has been proved in $[2,3]$, that the duality gap, which occurs by the presence of integrality, becomes small for a large number of units.

From now on we assume that the fuel costs are a piecewise linear function of the generated power and the start-up costs are given by (3.1). 
Let us associate the multipliers $\boldsymbol{\lambda}, \boldsymbol{\mu} \in \mathbb{R}^{T}$ with the demand and reserve-constraint respectively and consider the Lagrange function:

$$
\begin{gathered}
L(\boldsymbol{p}, \boldsymbol{u}, \boldsymbol{s}, \boldsymbol{w} ; \boldsymbol{\lambda}, \boldsymbol{\mu})=\sum_{t=1}^{T} \sum_{i=1}^{I} F C_{i}\left(\boldsymbol{p}_{i}^{t}, \boldsymbol{u}_{i}^{t}\right)+\sum_{t=1}^{T} \sum_{i=1}^{I} S C_{i}\left(\boldsymbol{u}_{i}(t)\right) \\
+\sum_{t=1}^{T}\left[\boldsymbol{\lambda}^{t}\left(D^{t}-\sum_{i=1}^{I} \boldsymbol{p}_{i}^{t}-\sum_{j=1}^{J}\left(\boldsymbol{s}_{j}^{t}-\boldsymbol{w}_{j}^{t}\right)\right)+\right. \\
\left.\boldsymbol{\mu}^{t}\left(R^{t}-\sum_{i=1}^{I}\left[\boldsymbol{u}_{i}^{t} p_{i}^{\max }-\boldsymbol{p}_{i}^{t}\right]\right)\right] .
\end{gathered}
$$

which has to be minimized subject to the constraints $(2.2)-(2.4)$ and $(2.7)-(2.9)$. We obtain the problem:

$$
\begin{aligned}
P(\boldsymbol{\lambda}, \boldsymbol{\mu}) \quad: \quad & \min L(\boldsymbol{p}, \boldsymbol{u}, \boldsymbol{s}, \boldsymbol{w} ; \boldsymbol{\lambda}, \boldsymbol{\mu}) \\
& \text { subject to }(2.2)-(2.4) \text { and }(2.7)-(2.9) .
\end{aligned}
$$

Denoting the marginal function of $P(\boldsymbol{\lambda}, \boldsymbol{\mu})$ by $d(\boldsymbol{\lambda}, \boldsymbol{\mu})$ the dual problem reads:

$$
\max \left\{d(\boldsymbol{\lambda}, \boldsymbol{\mu}): \boldsymbol{\lambda}, \boldsymbol{\mu} \in \mathbb{R}^{T}, \boldsymbol{\mu} \geq 0\right\},
$$

By the separability structure of $L(\boldsymbol{p}, \boldsymbol{u}, \boldsymbol{s}, \boldsymbol{w} ; \boldsymbol{\lambda}, \boldsymbol{\mu})$ and the constraints with respect to the units, we can decompose the problem $P(\boldsymbol{\lambda}, \boldsymbol{\mu})$ into problems $P_{i}(\boldsymbol{\lambda}, \boldsymbol{\mu})$ and $\tilde{P}_{j}(\boldsymbol{\lambda}, \boldsymbol{\mu})$ as follows:

$$
\begin{aligned}
P_{i}(\boldsymbol{\lambda}, \boldsymbol{\mu}): & \min _{\boldsymbol{u}_{i}} \sum_{t=1}^{T}\left[\min _{\boldsymbol{p}_{i}^{t}}\left\{F C_{i}\left(\boldsymbol{p}_{i}^{t}, \boldsymbol{u}_{i}^{t}\right)-\boldsymbol{\lambda}^{t} \boldsymbol{p}_{i}^{t}\right\}+S C_{i}\left(\boldsymbol{u}_{i}^{t}\right)-\boldsymbol{\mu}^{t} \boldsymbol{u}_{i}^{t} \boldsymbol{p}_{i}^{\max }\right] \\
& \text { subject to }(2.2) \text { and }(2.9)
\end{aligned}
$$

for the coal-fired and gas-burning units $(i=1, \ldots, I)$ and

$$
\begin{aligned}
\tilde{P}_{j}(\boldsymbol{\lambda}, \boldsymbol{\mu}): \quad \min _{\left(\boldsymbol{s}_{j}, \boldsymbol{w}_{j}\right)} \sum_{t=1}^{T}\left(\boldsymbol{\lambda}^{t}+\boldsymbol{\mu}^{t}\right)\left(\boldsymbol{w}_{j}^{t}-\boldsymbol{s}_{j}^{t}\right) \\
\quad \text { subject to }(2.3),(2.4),(2.7) \text { and }(2.8)
\end{aligned}
$$

for the pumped hydro storage plants $(j=1, \ldots, J)$. Denoting the marginal functions of the problems above by $d_{i}(\boldsymbol{\lambda}, \boldsymbol{\mu})$ and $\tilde{d}_{j}(\boldsymbol{\lambda}, \boldsymbol{\mu})$ respectively, we obtain for $d(\boldsymbol{\lambda}, \boldsymbol{\mu})$ :

$$
d(\boldsymbol{\lambda}, \boldsymbol{\mu})=\sum_{i=1}^{I} d_{i}(\boldsymbol{\lambda}, \boldsymbol{\mu})+\sum_{j=1}^{J} \tilde{d}_{j}(\boldsymbol{\lambda}, \boldsymbol{\mu})+\sum_{t=1}^{T}\left[\boldsymbol{\lambda}^{t} D^{t}+\boldsymbol{\mu}^{t} R^{t}\right]
$$

Useful properties of $d$ are its separability structure, concavity and the explicit formulas for computing subgradients. Setting $d^{\star}:=\max _{(\boldsymbol{\lambda}, \boldsymbol{\mu})} d(\boldsymbol{\lambda}, \boldsymbol{\mu})$ and $f_{\star}:=$ $\min _{(\boldsymbol{u}, \boldsymbol{p})} F(\boldsymbol{u}, \boldsymbol{p})$ it is known by the weak duality theorem that $d^{\star} \leq f_{\star}$. The relative duality gap $\left(f_{\star}-d^{\star}\right) / d^{\star}$ converges to zero as $I \longrightarrow \infty[2,3]$.

In order to solve the dual problem (4.3) efficiently, a fast non-smooth optimization method, a good initial guess for $(\boldsymbol{\lambda}, \boldsymbol{\mu})$, efficient algorithms for solving the subproblems (4.4) and (4.5) and a proper heuristics for computing a primal feasible solution are needed. We gave our preference to the bundle method described in $[8,9]$. 
The implemented algorithm works as follows.

- Initial guess for the Lagrange multipliers $\boldsymbol{\lambda}, \boldsymbol{\mu}$. Based on a priority list the on/off decisions are taken to satisfy the demand and reserve constraint for each time interval. Then the relative production costs of the on-line units are used to initialize $\boldsymbol{\lambda}$. $\boldsymbol{\mu}$ is set to be zero.

- Iterative procedure of the bundle method

- Computation of a value and of subgradients of $d(\boldsymbol{\lambda}, \boldsymbol{\mu})$ by solving $I+J$ subproblems of dimension $2 T$. The first $I$ problems are solved as follows. The minimization with respect to $\boldsymbol{p}_{i}^{t}$ is done explicitly or by onedimensional optimization. The minimization with respect to $\boldsymbol{u}_{i}$ is equivalent to the search for a shortest path in the state transition graph of the unit under consideration, and it is carried out by dynamic programming. Nodes passed during the minimal down time are not included in the state transition graph (cf [18]). The next $J$ hydro-storage subproblems are solved by the algorithm developed in [15].

- Bundle-method iteration.

- Determination of a primal feasible solution. The algorithm works in two steps to satisfy the (possibly violated) reserve constraints. First, we try to satisfy the constraints by using the pumped-storage hydro plants in those time intervals, where the largest values of $D^{t}+R^{t}$ occur. If the reserve-constraints are still violated, we modify the schedules of the thermal units by the procedure in [19]. The idea consists in finding a period t for which the reserve constraint is violated most and then computing the smallest amount of increase $\Delta \mu^{t}$ to satisfy the reserve constraint in this period. This procedure is carried out recursively until the constraints are satisfied for all periods.

- Economic dispatch. In a last step we improve the feasible solution found in the previous step. We solve the primal problem keeping the integer variables fixed. The latter linear optimization problem, referred to as economic dispatch, is solved using the CPLEX Callable Library [4].

The encouraging results of the test runs proved the efficiency of the dual approach. Computations based on the same data (model 1) as in the previous section provided the numerical results reported in Table 2 .

\begin{tabular}{|c|c|c|c|l|l|l|}
\hline \multirow{2}{*}{$\begin{array}{l}\text { NOA3 Optimality } \\
\text { tolerance: } 10^{-4}\end{array}$} & \multicolumn{2}{|c|}{ park 1 } & \multicolumn{2}{c|}{ park 2 } & \multicolumn{2}{c|}{ park 3 } \\
\cline { 2 - 7 } & time & gap & time & gap & time & gap \\
\hline \hline holiday week & $1: 36$ & $0.74 \%$ & $0: 40$ & $0.37 \%$ & $0: 35$ & $0.93 \%$ \\
\hline low load week & $0: 58$ & $1.47 \%$ & $0: 36$ & $0.23 \%$ & $0: 36$ & $0.54 \%$ \\
\hline peak load week & $0: 09$ & $-{ }^{1)}$ & $3: 43$ & $0.89 \%$ & $0: 39$ & $0.52 \%$ \\
\hline
\end{tabular}

1) No primal solution exists.

Table 2: CPU-time in minutes (HP-Apollo 735/125) and upper bound of the relative duality gap 


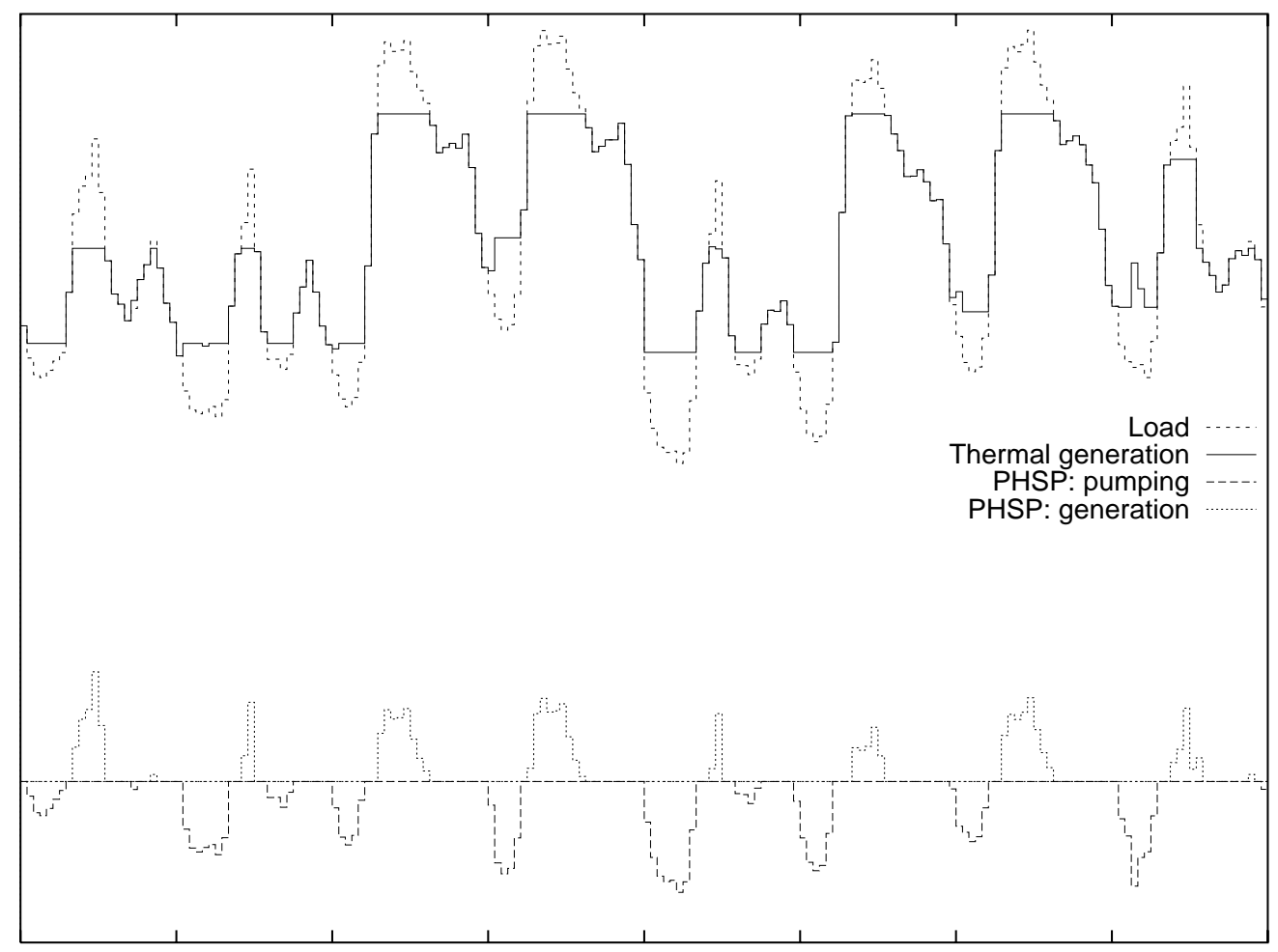

Fig. 2: Schedule for park 2 in the holiday week.

Acknowledgement. We wish to thank P. Reeh, G. Schwarzbach and J. Thomas (VEAG Vereinigte Energiewerke AG) for the outstanding collaboration which made the present paper possible.

\section{References}

[1] Aoki, K.; Itoh, M.; Satoh, T.; Nara, K.; Kanezashi, M.: Optimal Long-Term Unit Commitment in Large Scale Systems Including Fuel Constrained Thermal and Pumped-Storage Hydro. IEEE Transactions on Power Systems 4(1989), 1065 1073.

[2] Bertsekas, D.P.: Constrained Optimization and Lagrange Multiplier Methods. Academic Press, New York, 1982.

[3] Bertsekas, D.P.; Lauer, G.S.; Sandell, N.R.; Posbergh, T.A.: Optimal Short-Term Scheduling of Large-Scale Power Systems. IEEE Transactions on Automatic Control, AC-28(1983), 1-11.

[4] Using the CPLEX Callable Library. CPLEX Optimization, Inc. 1994.

[5] Dillon, T.S.; Edwin, K.W.; Kochs, H.-D.; Taud, R.J.: Integer Programming Approach to the Problem of Optimal Unit Commitment with Probabilistic Reserve Determination. IEEE Transactions on Power Apparatus and Systems 97(1978), $2154-2166$.

[6] Feltenmark, S.; Kiwiel, K.C.; Lindberg, P.-O.: Solving Unit Commitment Problems in Power Production Planning. Working Paper, 1996. 
[7] Guddat, J.; Römisch, W.; Schultz, R.: Some Applications of Mathematical Programming Techniques in Optimal Power Dispatch. Computing 49(1992), 193-200.

[8] Kiwiel, K.C.: Proximity Control in Bundle Methods for Convex Nondifferentiable Minimization. Mathematical Programming 46(1990), 105 - 122.

[9] Kiwiel, K.C.: User's Guide for NOA 2.0/3.0: A Fortran Package for Convex Nondifferentiable Optimization. Polish Academy of Science, System Research Institute, Warsaw, 1993/1994.

[10] Lemaréchal, C.; Pellegrino, F.; Renaud, A.; Sagastizábal, C.: Bundle Methods Applied to the Unit Commitment Problem. Proceedings of the 17th IFIP-Conference on System Modelling and Optimization, Prague, July 10 - 14, 1995. (to appear)

[11] Möller, A.: Über die Lösung des Blockauswahlproblems mittels Lagrangescher Relaxation. Diplomarbeit, Humboldt-Universität Berlin, Institut für Mathematik, 1994.

[12] Möller, A.; Römisch, W.: A Dual Method for the Unit Commitment Problem. Humboldt-Universität Berlin, Institut für Mathematik, Preprint Nr. 95-1, 1995.

[13] Muckstadt, J.A.; Koenig, S.A.: An Application of Lagrangian Relaxation to Scheduling in Thermal Power-Generation Systems. Operations Research, 25(1977), 387403.

[14] Müller, D.: Minimierung stückweise linearer Zielfunktionen mit Anwendung in der elektrischen Lastverteilung. Diplomarbeit, Humboldt-Universität Berlin, Institut für Mathematik, 1995.

[15] Nowak, M.: A Fast Descent Method for the Hydro Storage Subproblem in Power Generation. Working Paper, WP-96-109, IIASA, Laxenburg, 1996.

[16] van Roy, T.; Wolsey, L.A.: Valid Inequalities for Mixed 0-1 Programs. Discrete Applied Mathematics 14(1986), 199-213.

[17] Sheble, G.B.; Fahd, G.N.: Unit Commitment Literature Synopsis. IEEE Transactions on Power Systems 9(1994), 128-135.

[18] Takriti, S.; Birge, J.R.; Long, E.: A Stochastic Model for the Unit Commitment Problem. IEEE Transactions on Power Systems. (to appear)

[19] Zhuang, F.; Galiana, F.D.: Towards a More Rigorous and Practical Unit Commitment by Lagrangian Relaxation. IEEE Transactions on Power Systems 3(1988), $763-773$. 